\section{MACHINES UNIPOLAIRES INDUSTRIELLES}

On sait que l'on a donné aux machines basées sur le principe bien connu du disque de Faraday le nom assez impropre de dynamos unipolaires, par opposition aux machines bi ou multipolaires, dans l'induit desquelles la force électromotrice change périodiquement de sens et qui exigent un commutateur redresseur spécial pour fournir des courants de direction invariable.

Le disque de Faraday est en réalité une machine d'induction d'une très grande simplicité, c'est la dynamo la plus simple connue jusqu'à présent et qui fournit une force électromotrice rigoureusement constante lorsque la vitesse de rotation est ellemême constante. En appuyant un balai sur l'axe et un autre sur la périphérie du disque on obtient un courant constant sans l'aide d'aucun collecteur qui, comme on le sait, constitue l'une des pièces délicates des dynamos à plusieurs pôles. De plus, le flux magnétique traversant l'induit (le disque) toujours dans le même sens n'est jamais inversé, et il n'y a par suite pas de pertes par hystérésis dans ce genre de machines. Quant aux courants de Foucault, ce sont justement eux qu'on utilise. Les avantages de cet appareil sont donc remarquables.

Cependant, par leur constitution même, ces machines ne peuvent produire, avec les vitesses ordinairement employées, qu'une force électromotrice très faible. Aussi, dans l'industrie, n'ont-elles guère été employées qu'à la production de courants très intenses, sous de très faibles voltages, tels qu'en demandent certaines applications électrolytiques.

On a bien essayé d'employer plusieurs disques isolés les uns des autres et reliés électriquement en série, mais les résultats n'ont pas été favorables. Au lieu d'augmenter le nombre de disques on peut augmenter le nombre de tours: mais tant que l'on a eu que les machines à vapeur à mouvement alternatif, le

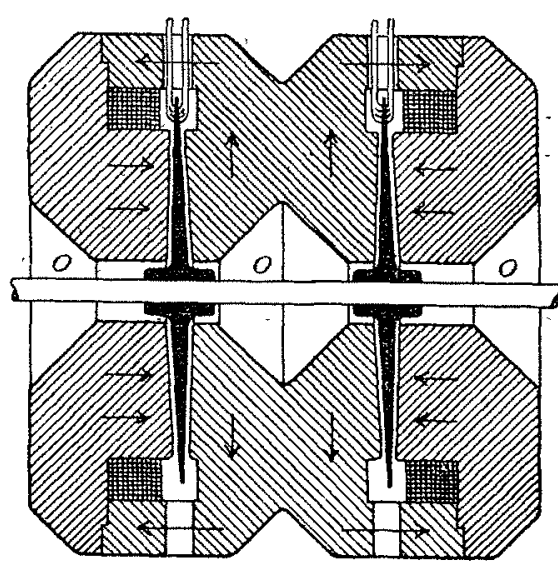
nombre de tours maximum réalisable pratiquement était encore beaucoup trop faible; maintenant que l'on dispose de turbines à vapeur tournant à des vitesses inconnues jusqu'ici il semble que l'avantage doit revenir aux machines unipolaires.

M. Seidener, dans les Zeitschrift fur Electrotechnili de Vienne, s'est attaché à cette question de l'entraînement des dynamos unipolaires par des Schéma d'une dynamo unipolarre double. turbines à vapeur, et il est convaincu que ces groupes électrogènes peuvent donner de très bons résultats.

Cet auteur propose le type de machine suivant, dont nous reproduisons ici un schéma. Sur un même arbre sont calés deux disques parfaitement équilibrés et tournant dans des cavités ménagées à l'intérieur d'une carcasse magnétique. La simplicité de la machine permet de se rendre immédiatement compte de son fonctionnement.

Dans le cas de la figure ci-contre, deux balais appuyent à la périphérie des disques et les champs magnétiques sont tels que les courants induits par la rotation commune vont, dans ces disques, de la périphérie au centre pour l'un, et du centre à la périphérie pour l'autre. Les deux disques sont en série et le voltage total est le double de ce qu'il serait avec un disque seul.

Si l'on inverse le champ magnétique de l'un des disques, les courants ont alors le même sens dans les deux roues; les deux frotteurs précédents fonctionnent en parallèle et si l'on dispose un troisieme frotteur sur l'axe de rotation on constitue un circuit fermé. La différence de potentiel est alors la moitié de celle du premier cas.
En adoptant la première disposition des champs magnétiques et en installant un frotteur sur 1'axe, entre les deux disques, on réalise le montage d'une distribution à trois fils; le fil neutre aboutissant au balai qui frotte sur l'axe.

Un calcul simple permet de montrer que ces machines peuvent produire de 50 à 500 volts avec des vitesses de rotation de 1500 . à 12 ooo tours par minute.

Pour des considérations d'ordre mécanique, l'auteur recom. mande de capter le courant, non pas à la périphérie proprement dite mais sur les bords latéraux.

\section{TRANSPORT D'ÉNERGIE DE GROMO}

\section{A 40000 volTs}

L'installation suivante a été établie en vue d'utiliser, de suite, une puissance de 2000 chevaux au moyen d'une usiné hydro-électrique située à Gromo dans la vallée du Sério, et, ultérieurement, un supplément de 2,000 chevaux, au moyén d'une autre usine située un peu plus haut.

Toute l'énergie doit être transportée de Gromo à Nembro, par courants truphasés au moyen de deux lignes posées sur leł mêmes poteaux, avec faculté de pouvoir transporter l'énérgie au delà de Nembro avec une perte en ligne aussi faible que possıble, tout en visant à l'économie dans les frais de premièrè installation.

Telles étaient les conditions du problème. Voyons comment l'on y a satisfait, et quelles ont été les raisons qui ont décidé la maison Brown Boveri \& $C^{i e}$ de Baden, qui a faif toute l'installation, à adopter une tension aussi élevée pour-uñ transport d'énergie de grandeur moyenne, et à une distance relativement faible, car celle-ci n'est que de 32 kilomètres.

Dans l'établissement du premier projet on avait prévu : tué tension de 30 ooo volts, une ligne composée de 3 fils en cuivte de $6,5 \mathrm{~mm}$. de diamètre, et une perte en ligne d'environ 4 p. 100 pour le transport de 2000 chevaux. Ces données correspon daient en effet, pour cette unstallation, à des conditions tres avantageuses qu'on n'aurait pu réaliser avec une tension plüs basse. Pour les 2000 chevaux à installer plus tard, on aurall monté sur les mêmes poteaux une deuxième ligne.

Cependant, le montage de cette deuxième ligne, sans inter. rompre le service de l'installation, aurait présenté certaine:

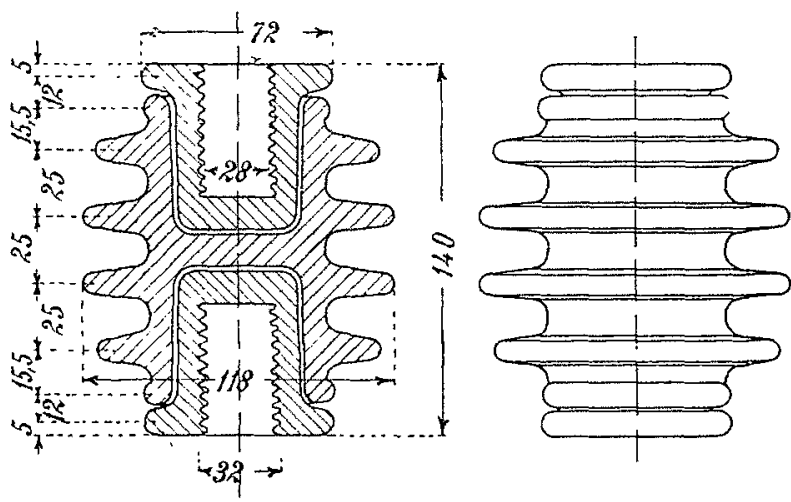

Fig. 1. - Isolateur employé à l'intérieur pour les connections à 40000 volts.

ment des difficultés, surtout pour le cas spécial qui nous occupe. En outre si, à première vue, on peut considérer l'unt des lignes comme réserve de l'autre, cet avantage ne se manifeste guère en pratıque que si les deux lignes sont montées sur deux rangées de poteaux distinctes.

Cette dernière solution écartée, à cause des frais qu'elle entraînait, il était préférable de prévoir, dès le début, unt ligne capable, à elle seule, de transporter les 4000 chevátxi 